\title{
Assessment of the soil quality of Haridwar Uttarakhand India: A comparative study
}

\author{
Bhardwaj S. ${ }^{1}$, Khanna D.R. ${ }^{2}$, Ruhela M. ${ }^{1}$, Bhutiani R. ${ }^{2}$, Bhardwaj R. ${ }^{4}$ and Ahamad F. ${ }^{3}$ 凶
}

Received: 10.08.2020

Revised: 01.11.2020

Accepted: 23.11.2020

\begin{abstract}
The present study aims to compare the quality of soil of different region of Haridwar with reference to physicochemical and heavy metal parameters. To fulfill the objectives of present study, soil sampling was performed in forest (control site), industrial, residential and agricultural areas in and around Haridwar. Soil samples were analyzed for different physicochemical and heavy metal parameters. Values of all the studied soil parameters were found highest (an increase of $32 \%$ in temperature $\left(16.63\right.$ to $\left.21.64^{\circ} \mathrm{C}\right), 121 \%$ in soil moisture $(13.05$ to $28.39 \%), 29.02 \%$ in soil porosity $(37.56$ to $49.03 \%)$, and $19.6 \%$ in the water holding capacity (36.22 to $43.58 \%), 74.18 \%$ in conductivity $(0.25$ to $0.40 \mu M h o s / c m)$, and $203.78 \%$ in chloride $(16.67$ to $53.97 \mathrm{mg} / \mathrm{gm})$ ) at the industrial area in comparison to other sites. During the course of the study, an increasing trend in all the parameters at all the sites was observed this may be due to the dumping of industrial solid waste and effluent. Although no negative impact was observed on the soil quality but continuous dumping will results in harmful impacts due to the accumulation of pollutants. Therefore there is a need for safe and proper disposal and utilization techniques to manage the enormous quantity of industrial waste. All the heavy metals (such as copper $(0.050$ to $0.055 \mathrm{mg} / \mathrm{gm})$, manganese $(0.232$ to $0.242 \mathrm{mg} / \mathrm{gm})$, nickel $(0.035$ to $0.036 \mathrm{mg} / \mathrm{gm})$, lead $(0.039 \mathrm{mg} / \mathrm{gm})$, and iron (1.19 to 1.22) were found in higher concentration during the study period while cadmium was found absent during the study period.
\end{abstract}

Key Words: Accumulation, Soil porosity, Threshold value, Water holding capacity

\section{Introduction}

Soil is a thin veneer that covers most of the earth's surface. Different natural forces acting on natural material result in the formation of upper layer of the soil; it is classified into different horizons based on morphology, physical and chemical properties, composition, and biological characteristics (James et al., 2014). In most cases, solid waste added to soil primarily affect chemical properties of soil such as $\mathrm{pH}$ and fertility and it depends on the loading rates of dumps. With the increasing population and industrial growth, the need for power has increased manifold (Raja et al., 2015). For the last two decades, the rapid growth of

\section{Author's Address}

${ }^{1}$ Department of Environmental Engineering, Subharti Institute of Technology and Engineering (SITE), Swami Vivekanand Subharti University, Meerut-250005, (UP) India

${ }^{2}$ Department of Zoology and Environmental Sciences, Gurukula Kangri Vishwavidyalaya, Haridwar-249404, (UK) India

${ }^{3}$ Co Gen department, Nanglmal Sugar complex Meerut

${ }^{3}$ Department of Environmental Science, Keral Verma Subharti

College of Sciences (KVSCOS), Swami Vivekanand Subharti

University, Meerut-250005, (UP) India

E-mail.: faheem.ahamad170390@gmail.com industrialization and urbanization has created a negative impact on the environment which is due to industrial, municipal, and agricultural wastes having a large number of pesticides, insecticides, fertilizer residues, fly ash, heavy metals, etc. In the $21^{\text {st }}$ century, practices such as agriculture, mining, logging, housing, solid waste dumping and wastewater discharge increased to such an extent that their impacts in forms of deteriorated air quality, soil quality, loss of biodiversity, and water quality can be seen (Bharti and Kamboj, 2018). pH of soil, concentration of organic matter, conductivity and valency of ions affects the accessibility of soil nutrients to plants (Jiang et al., 2009). Physical, chemical and micro-biological properties along with interaction of microbial flora and fauna present in the soil affect the quality of soil (Papendick and Parr, 1992). Due to less availability of fresh water and deep water table, farmers show their increased interest in irrigation with partially treated or untreated wastewater, which is a threat to the soil quality (Bougnom et al., 2020; Zhang and Shen, 2019). Irrigation with wastewater increased the amount of pollutants in 
slowly by the process of accumulation. These accumulated pollutants when transferred in the tissues of plants affects the plants and as well as crops and forest ecosystem to a large extent (Courault et al., 2017; Kumar et al., 2019; Sarwar et al., 2019). Although irrigation with wastewater is a sustainable way but it needs utmost for the protection of soil quality. The Soil must be managed and protected carefully for the most beneficial use in the future therefore the present study is aimed to assess soil quality affected by the disposal of industrial waste in different areas of Haridwar. In the present study a comparison of soil of forest area, industrial area, residential area, and agricultural area was performed.

\section{Materials and Methods}

For the present study, soil samples were collected monthly from different sampling sites during January 2013 to December 2014 in morning hours. The samples were taken in polyethene from each location. The analysis of soil samples were performed following the standard methods of Singh et al. (1999) and Trivedi and Goel (1986). Soil profile of Haridwar region is given in figure 1 (http://agricoop.nic.in/sites/default/files/haridwar.p df).

\section{Soil sampling sites Industrial area Haridwar}

a) Forest areas (SS1) - The sample were collected from the forest area of Haridwar. This site is considered as control site.

b) Residential area (SS2) - This site is the residential area of Haridwar. Sampling was performed at two points and then a composite sample was prepared.

c) Industrial area (SS3) - This site is situated near the SIDCUL area Haridwar. Sampling was performed at three points and then a composite sample was prepared.

d) Agricultural field (SS4) - This site is agricultural area located around Haridwar city. Sampling was performed at five points and then a composite sample was prepared.

Figure 1. Soil profile of Haridwar region (http://agricoop.nic.in/sites/default/files/haridwar.pdf)

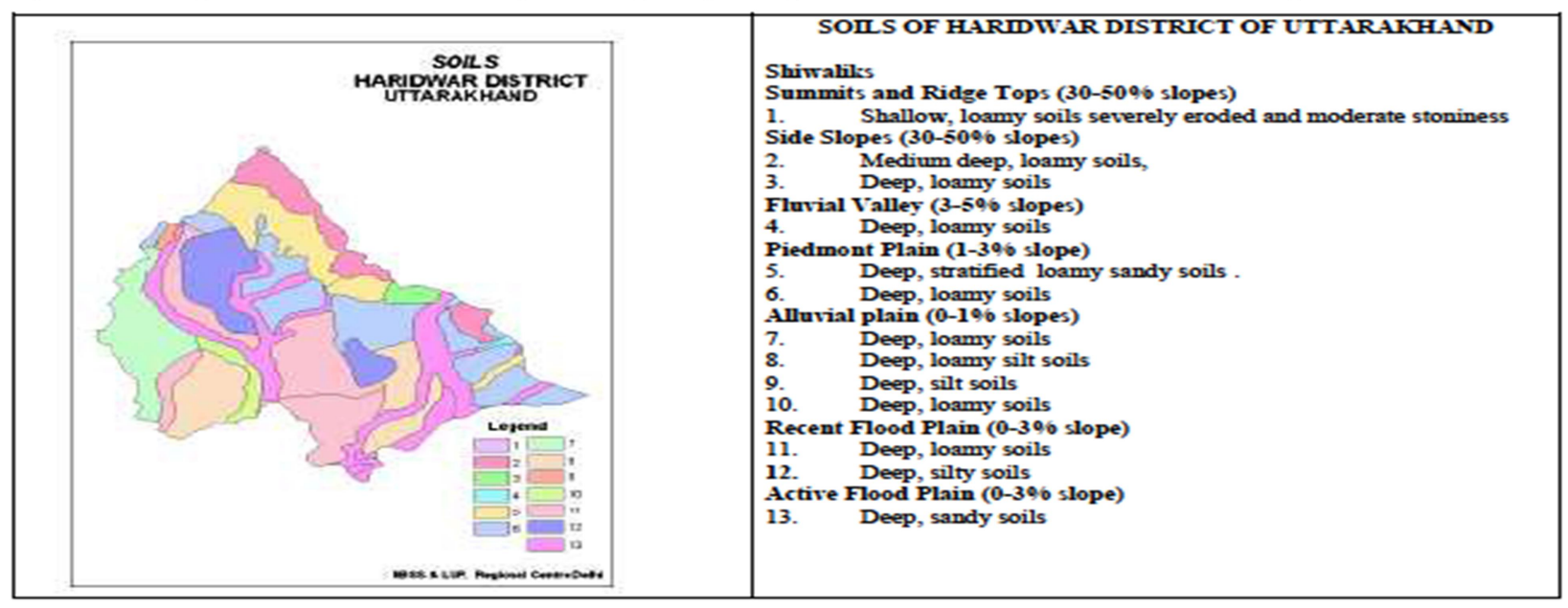

\section{Results and Discussion}

The Physicochemical parameters of the soil of all the four sites are presented in the Table 1 to 8 for both the year (2013 and 2014) and the average values are given in table 9. During the whole study period, minimum average temperature was found $16.42^{\circ} \mathrm{C} \pm 1.79$ for the first year in the soil of the Control site (SS1) while maximum average temperature was found $21.75^{\circ} \mathrm{C} \pm 0.74$ for the second year in the soil of the Industrial area (SS3) and the average values of temperature was found $16.63^{\circ} \mathrm{C}$ $\pm 0.30,18.93^{\circ} \mathrm{C} \pm 0.11,21.64^{\circ} \mathrm{C} \pm 0.16$, and $20.55^{\circ} \mathrm{C}$ \pm 0.83 at SS1, SS2, SS3, and SS4 respectively. Temperature was found maximum at the industrial area site $\left(21.64^{0} \mathrm{C} \pm 0.16\right)$ while minimum was found at the control site $\left(16.63^{\circ} \mathrm{C} \pm 0.30\right)$. In literature no study was found regarding temperature change in the soil. During the whole study period, minimum average $\mathrm{pH}$ was found $7.53 \pm 0.45$ for the first year 
Table 1. Study of physicochemical parameters of Soil at Control site (Forest area) during 2013-2014

\begin{tabular}{|c|c|c|c|c|c|c|c|c|c|c|c|c|c|c|c|}
\hline \multirow{3}{*}{$\underset{\downarrow}{\stackrel{3}{\Xi}}$} & \multirow{3}{*}{$\begin{array}{l}\text { Year } \\
\rightarrow\end{array}$} & \multicolumn{14}{|c|}{ Parameters } \\
\hline & & \multicolumn{2}{|c|}{ Temperature $\left({ }^{\circ} \mathrm{C}\right)$} & \multicolumn{2}{|l|}{$\mathbf{p H}$} & \multicolumn{2}{|c|}{ Soil Moisture (\%) } & \multicolumn{2}{|c|}{ Porosity (\%) } & \multicolumn{2}{|c|}{$\begin{array}{l}\text { Water Holding } \\
\text { Capacity (\%) }\end{array}$} & \multicolumn{2}{|c|}{$\begin{array}{l}\text { Conductivity } \\
(\mu \mathrm{Mhos} / \mathrm{cm})\end{array}$} & \multicolumn{2}{|c|}{ Chloride (mg/gm) } \\
\hline & & 2013 & 2014 & 2013 & 2014 & 2013 & 2014 & 2013 & 2014 & 2013 & 2014 & 2013 & 2014 & 2013 & 2014 \\
\hline \multicolumn{2}{|c|}{ Nov } & 14.3 & 14.8 & 7.2 & 7.3 & 11.26 & 11.07 & 37.8 & 38.5 & 35.7 & 35.6 & 0.208 & 0.277 & 15.12 & 15.27 \\
\hline \multicolumn{2}{|c|}{ Dec } & 14.2 & 13.9 & 7.3 & 7.7 & 11.02 & 10.87 & 38.5 & 39.6 & 35.6 & 34.3 & 0.176 & 0.218 & 15.07 & 14.97 \\
\hline \multicolumn{2}{|c|}{ Jan } & 13.7 & 13.2 & 7.1 & 6.9 & 12.02 & 11.35 & 39.6 & 37.5 & 34.5 & 36.6 & 0.218 & 0.247 & 14.90 & 15.54 \\
\hline \multicolumn{2}{|c|}{ Feb } & 14.0 & 13.8 & 6.9 & 7.0 & 12.72 & 12.07 & 37.5 & 34.9 & 36.6 & 37.2 & 0.231 & 0.234 & 15.41 & 16.87 \\
\hline \multicolumn{2}{|c|}{ Mar } & 17.0 & 17.7 & 7.2 & 7.3 & 13.00 & 12.89 & 34.9 & 36.4 & 37.2 & 37.4 & 0.234 & 0.254 & 16.86 & 17.01 \\
\hline \multicolumn{2}{|c|}{ Apr } & 17.1 & 18.5 & 7.1 & 7.7 & 14.25 & 13.74 & 36.4 & 34.7 & 36.2 & 34.7 & 0.254 & 0.258 & 17.01 & 17.46 \\
\hline \multicolumn{2}{|c|}{ May } & 18.0 & 18.7 & 7.6 & 8.3 & 14.84 & 14.26 & 34.7 & 35.6 & 36.7 & 38.9 & 0.247 & 0.270 & 17.45 & 18.08 \\
\hline \multicolumn{2}{|c|}{ Jun } & 18.2 & 18.5 & 8.1 & 8.2 & 14.99 & 14.77 & 35.6 & 36.2 & 38.9 & 36.8 & 0.270 & 0.256 & 18.09 & 18.17 \\
\hline \multicolumn{2}{|c|}{ Jul } & 18.2 & 18.6 & 8.0 & 8.1 & 14.86 & 14.54 & 36.2 & 34.7 & 36.8 & 37.8 & 0.256 & 0.274 & 17.58 & 17.54 \\
\hline \multicolumn{2}{|c|}{ Aug } & 17.5 & 18.9 & 8.1 & 8.0 & 13.74 & 13.96 & 34.7 & 36.4 & 37.8 & 34.8 & 0.274 & 0.278 & 16.97 & 17.72 \\
\hline \multicolumn{2}{|c|}{ Sep } & 17.5 & 17.7 & 7.9 & 7.8 & 12.86 & 13.12 & 36.4 & 37.1 & 36.5 & 39.0 & 0.278 & 0.281 & 16.80 & 17.14 \\
\hline \multicolumn{2}{|c|}{ Oct } & 17.3 & 17.8 & 7.9 & 7.6 & 12.15 & 12.74 & 37.1 & 37.8 & 39.4 & 34.7 & 0.281 & 0.208 & 16.41 & 16.63 \\
\hline \multicolumn{2}{|c|}{$\begin{array}{l}\text { Average } \\
\pm \text { SD }\end{array}$} & $\begin{array}{l}16.42 \\
\pm 1.79 \\
\end{array}$ & $\begin{array}{l}16.84 \\
\pm 2.22 \\
\end{array}$ & $\begin{array}{l}7.53 \\
\pm 0.45 \\
\end{array}$ & $\begin{array}{l}7.66 \\
\pm 0.46 \\
\end{array}$ & $\begin{array}{l}13.14 \\
\pm 1.39 \\
\end{array}$ & $\begin{array}{l}12.95 \\
\pm 1.36 \\
\end{array}$ & $\begin{array}{l}36.62 \\
\pm 1.55 \\
\end{array}$ & $\begin{array}{l}38.5 \\
\pm 1.52 \\
\end{array}$ & $\begin{array}{l}36.83 \\
\pm 1.37 \\
\end{array}$ & $\begin{array}{l}35.6 \\
\pm 1.704 \\
\end{array}$ & $\begin{array}{l}0.244 \\
\pm 0.032 \\
\end{array}$ & $\begin{array}{l}0.255 \\
\pm 0.024 \\
\end{array}$ & $\begin{array}{l}16.47 \\
\pm 1.09 \\
\end{array}$ & $\begin{array}{l}16.87 \\
\pm 1.08 \\
\end{array}$ \\
\hline
\end{tabular}

Table 2. Heavy metals analysis of Soil at Control site (Forest area) during 2013-2014

\begin{tabular}{|c|c|c|c|c|c|c|c|c|c|c|c|c|c|c|c|}
\hline \multirow{3}{*}{ 方 } & \multirow{3}{*}{$\begin{array}{l}\text { Year } \\
\rightarrow\end{array}$} & \multicolumn{14}{|c|}{ Parameters } \\
\hline & & \multicolumn{2}{|c|}{$\mathrm{Cu}$ (mg/gm) } & \multicolumn{2}{|c|}{$\mathrm{Cd}(\mathrm{mg} / \mathrm{gm})$} & \multicolumn{2}{|c|}{ Mn (mg/gm) } & \multicolumn{2}{|c|}{$\mathrm{Ni}(\mathrm{mg} / \mathrm{gm})$} & \multicolumn{2}{|c|}{ Pb (mg/gm) } & \multicolumn{2}{|c|}{$\mathrm{Cr}^{+6}(\mathrm{mg} / \mathrm{gm})$} & \multicolumn{2}{|c|}{$\mathrm{Fe}(\mathrm{mg} / \mathrm{gm})$} \\
\hline & & 2013 & 2014 & 2013 & 2014 & 2013 & 2014 & 2013 & 2014 & 2013 & 2014 & 2013 & 2014 & 2013 & 2014 \\
\hline \multicolumn{2}{|l|}{ Nov } & 0.037 & 0.041 & 0.00 & 0.00 & 0.243 & 0.245 & 0.036 & 0.035 & 0.033 & 0.036 & 0.040 & 0.043 & 1.96 & 1.98 \\
\hline \multicolumn{2}{|l|}{ Dec } & 0.032 & 0.035 & 0.00 & 0.00 & 0.233 & 0.236 & 0.033 & 0.036 & 0.032 & 0.035 & 0.038 & 0.039 & 1.95 & 1.98 \\
\hline \multicolumn{2}{|c|}{ Jan } & 0.030 & 0.032 & 0.00 & 0.00 & 0.246 & 0.245 & 0.027 & 0.035 & 0.031 & 0.035 & 0.035 & 0.038 & 1.89 & 1.99 \\
\hline \multicolumn{2}{|l|}{ Feb } & 0.034 & 0.035 & 0.00 & 0.00 & 0.267 & 0.258 & 0.025 & 0.036 & 0.030 & 0.036 & 0.038 & 0.042 & 1.92 & 1.93 \\
\hline \multicolumn{2}{|c|}{ Mar } & 0.030 & 0.033 & 0.00 & 0.00 & 0.212 & 0.241 & 0.034 & 0.038 & 0.028 & 0.031 & 0.032 & 0.037 & 1.89 & 1.88 \\
\hline \multicolumn{2}{|l|}{ Apr } & 0.025 & 0.030 & 0.00 & 0.00 & 0.226 & 0.230 & 0.026 & 0.029 & 0.029 & 0.031 & 0.026 & 0.029 & 1.79 & 1.88 \\
\hline \multicolumn{2}{|c|}{ May } & 0.027 & 0.028 & 0.00 & 0.00 & 0.247 & 0.245 & 0.030 & 0.032 & 0.030 & 0.032 & 0.025 & 0.028 & 1.95 & 1.92 \\
\hline \multicolumn{2}{|c|}{ Jun } & 0.031 & 0.030 & 0.00 & 0.00 & 0.217 & 0.227 & 0.032 & 0.030 & 0.032 & 0.035 & 0.035 & 0.034 & 1.79 & 1.88 \\
\hline \multicolumn{2}{|l|}{ Jul } & 0.034 & 0.038 & 0.00 & 0.00 & 0.234 & 0.236 & 0.025 & 0.032 & 0.030 & 0.033 & 0.034 & 0.038 & 1.87 & 1.88 \\
\hline \multicolumn{2}{|c|}{ Aug } & 0.033 & 0.035 & 0.00 & 0.00 & 0.220 & 0.231 & 0.023 & 0.029 & 0.027 & 0.035 & 0.032 & 0.034 & 1.96 & 1.97 \\
\hline \multicolumn{2}{|l|}{ Sep } & 0.032 & 0.033 & 0.00 & 0.00 & 0.222 & 0.235 & 0.027 & 0.034 & 0.029 & 0.032 & 0.031 & 0.034 & 1.87 & 1.89 \\
\hline \multicolumn{2}{|l|}{ Oct } & 0.027 & 0.030 & 0.00 & 0.00 & 0.241 & 0.244 & 0.040 & 0.041 & 0.030 & 0.036 & 0.034 & 0.038 & 2.07 & 1.98 \\
\hline \multicolumn{2}{|c|}{$\begin{array}{l}\text { Average } \\
\pm \text { SD }\end{array}$} & $\begin{array}{l}0.031 \\
\pm 0.003 \\
\end{array}$ & $\begin{array}{l}\mathbf{0 . 0 3 3} \\
\pm 0.004 \\
\end{array}$ & $\begin{array}{l}\mathbf{0 . 0 0} \\
\pm 0.00 \\
\end{array}$ & $\begin{array}{l}\mathbf{0 . 0 0} \\
\pm 0.00 \\
\end{array}$ & $\begin{array}{l}0.234 \\
\pm 0.016 \\
\end{array}$ & $\begin{array}{l}0.239 \\
\pm 0.009 \\
\end{array}$ & $\begin{array}{l}\mathbf{0 . 0 3 0} \\
\pm 0.005 \\
\end{array}$ & $\begin{array}{l}0.034 \\
\pm 0.004 \\
\end{array}$ & $\begin{array}{l}0.030 \\
\pm 0.002 \\
\end{array}$ & $\begin{array}{l}0.034 \\
\pm 0.002 \\
\end{array}$ & $\begin{array}{l}\mathbf{0 . 0 3 3} \\
\pm 0.005 \\
\end{array}$ & $\begin{array}{l}0.036 \\
\pm 0.004 \\
\end{array}$ & $\begin{array}{l}1.91 \\
\pm 0.077 \\
\end{array}$ & $\begin{array}{l}1.93 \\
\pm 0.047 \\
\end{array}$ \\
\hline
\end{tabular}


Bhardwaj et al.

Table 3. Study of physicochemical parameters of Soil at the residential area site during 2013-2014

\begin{tabular}{|c|c|c|c|c|c|c|c|c|c|c|c|c|c|c|c|}
\hline \multirow{3}{*}{$\underset{\mathfrak{\downarrow}}{\stackrel{3}{\Xi}}$} & \multirow{3}{*}{$\begin{array}{l}\text { Year } \\
\rightarrow\end{array}$} & \multicolumn{14}{|c|}{ Parameters } \\
\hline & & \multicolumn{2}{|c|}{ Temperature $\left({ }^{\circ} \mathrm{C}\right)$} & \multicolumn{2}{|l|}{ pH } & \multicolumn{2}{|c|}{ Soil Moisture (\%) } & \multicolumn{2}{|c|}{ Porosity (\%) } & \multicolumn{2}{|c|}{$\begin{array}{l}\text { Water Holding } \\
\text { Capacity (\%) }\end{array}$} & \multicolumn{2}{|c|}{$\begin{array}{l}\text { Conductivity } \\
\text { ( } \mu \text { Mhos/cm) }\end{array}$} & \multicolumn{2}{|c|}{ Chloride (mg/gm) } \\
\hline & & 2013 & 2014 & 2013 & 2014 & 2013 & 2014 & 2013 & 2014 & 2013 & 2014 & 2013 & 2014 & 2013 & 2014 \\
\hline \multicolumn{2}{|c|}{ Nov } & 18.6 & 18.5 & 7.4 & 7.8 & 15.61 & 15.87 & 38.3 & 40.5 & 39.2 & 41.5 & 0.208 & 0.218 & 16.08 & 16.17 \\
\hline \multicolumn{2}{|l|}{ Dec } & 18.3 & 18.2 & 7.3 & 7.5 & 15.32 & 16.34 & 38.5 & 39.7 & 38.8 & 40.2 & 0.177 & 0.197 & 16.75 & 16.56 \\
\hline \multicolumn{2}{|l|}{ Jan } & 18.1 & 18.3 & 7.4 & 7.6 & 16.19 & 16.86 & 36.9 & 38.6 & 37.4 & 39.2 & 0.168 & 0.175 & 16.84 & 16.78 \\
\hline \multicolumn{2}{|l|}{ Feb } & 18.5 & 18.4 & 7.4 & 7.3 & 16.46 & 17.33 & 37.2 & 38.2 & 36.8 & 37.6 & 0.157 & 0.167 & 16.90 & 16.86 \\
\hline \multicolumn{2}{|c|}{ Mar } & 18.7 & 18.7 & 7.5 & 7.5 & 17.06 & 17.71 & 38.2 & 39.4 & 38.6 & 39.3 & 0.167 & 0.182 & 16.94 & 17.16 \\
\hline \multicolumn{2}{|c|}{ Apr } & 19.1 & 19.4 & 7.3 & 7.4 & 17.34 & 18.06 & 39.2 & 41.2 & 40.1 & 41.4 & 0.173 & 0.194 & 17.04 & 17.64 \\
\hline \multicolumn{2}{|c|}{ May } & 19.2 & 19.8 & 7.6 & 7.8 & 18.23 & 18.54 & 37.8 & 39.2 & 39.8 & 41.1 & 0.178 & 0.238 & 17.25 & 18.07 \\
\hline \multicolumn{2}{|l|}{ Jun } & 19.5 & 20.1 & 6.9 & 7.3 & 18.74 & 18.89 & 38.2 & 38.7 & 39.7 & 40.7 & 0.174 & 0.224 & 17.78 & 18.25 \\
\hline \multicolumn{2}{|l|}{ Jul } & 20.0 & 20.0 & 8.1 & 8.0 & 19.19 & 18.79 & 34.7 & 35.8 & 40.2 & 42.5 & 0.210 & 0.215 & 18.06 & 17.89 \\
\hline \multicolumn{2}{|c|}{ Aug } & 19.5 & 19.7 & 8.4 & 8.5 & 18.32 & 17.87 & 38.9 & 38.5 & 41.2 & 43.5 & 0.230 & 0.235 & 17.84 & 17.53 \\
\hline \multicolumn{2}{|l|}{ Sep } & 18.0 & 18.1 & 7.8 & 7.9 & 17.79 & 17.14 & 39.4 & 39.8 & 41.4 & 42.6 & 0.225 & 0.232 & 17.31 & 17.17 \\
\hline \multicolumn{2}{|l|}{ Oct } & 18.7 & 18.3 & 8.1 & 8.4 & 16.45 & 16.72 & 37.4 & 39.6 & 40.4 & 41.3 & 0.236 & 0.230 & 16.85 & 16.91 \\
\hline \multicolumn{2}{|c|}{$\begin{array}{l}\text { Average } \\
\pm \text { SD }\end{array}$} & $\begin{array}{l}18.85 \\
\pm 0.62\end{array}$ & $\begin{array}{l}19.0 \\
\pm 0.78\end{array}$ & $\begin{array}{l}7.56 \\
\pm 0.419 \\
\end{array}$ & $\begin{array}{l}7.75 \\
\pm 0.39 \\
\end{array}$ & $\begin{array}{l}17.83 \\
\pm 1.25\end{array}$ & $\begin{array}{l}17.49 \\
\pm 1.01 \\
\end{array}$ & $\begin{array}{l}37.89 \\
\pm 1.266 \\
\end{array}$ & $\begin{array}{l}39.10 \\
\pm 1.35 \\
\end{array}$ & $\begin{array}{l}39.47 \\
\pm 1.394\end{array}$ & $\begin{array}{l}40.91 \\
\pm 1.65 \\
\end{array}$ & $\begin{array}{l}\mathbf{0 . 1 9 2} \\
\pm 0.028 \\
\end{array}$ & $\begin{array}{l}0.209 \\
\pm 0.025\end{array}$ & $\begin{array}{l}17.14 \\
\pm 0.55\end{array}$ & $\begin{array}{l}17.25 \\
\pm 0.64 \\
\end{array}$ \\
\hline
\end{tabular}

Table 4. Heavy metals analysis of Soil of at the residential area site during 2013-2014

\begin{tabular}{|c|c|c|c|c|c|c|c|c|c|c|c|c|c|c|c|}
\hline \multirow{3}{*}{$\stackrel{3}{\Xi}$} & \multirow{3}{*}{$\begin{array}{l}\text { Year } \\
\rightarrow\end{array}$} & \multicolumn{14}{|c|}{ Parameters } \\
\hline & & \multicolumn{2}{|c|}{$\mathrm{Cu}$ (mg/gm) } & \multicolumn{2}{|c|}{ Cd (mg/gm) } & \multicolumn{2}{|c|}{ Mn (mg/gm) } & \multicolumn{2}{|c|}{$\mathrm{Ni}(\mathrm{mg} / \mathrm{gm})$} & \multicolumn{2}{|c|}{$\mathrm{Pb}$ (mg/gm) } & \multicolumn{2}{|c|}{$\mathrm{Cr}^{+6}(\mathrm{mg} / \mathrm{gm})$} & \multicolumn{2}{|c|}{$\mathrm{Fe}(\mathrm{mg} / \mathrm{gm})$} \\
\hline & & 2013 & 2014 & 2013 & 2014 & 2013 & 2014 & 2013 & 2014 & 2013 & 2014 & 2013 & 2014 & 2013 & 2014 \\
\hline \multicolumn{2}{|c|}{ Nov } & 0.050 & 0.057 & 0.00 & 0.00 & 0.245 & 0.231 & 0.038 & 0.044 & 0.037 & 0.039 & 0.057 & 0.051 & 0.980 & 0.920 \\
\hline \multicolumn{2}{|c|}{ Dec } & 0.045 & 0.051 & 0.00 & 0.00 & 0.243 & 0.234 & 0.027 & 0.036 & 0.035 & 0.041 & 0.053 & 0.046 & 1.032 & 0.997 \\
\hline \multicolumn{2}{|c|}{ Jan } & 0.040 & 0.048 & 0.00 & 0.00 & 0.231 & 0.225 & 0.036 & 0.032 & 0.028 & 0.032 & 0.051 & 0.044 & 1.075 & 0.984 \\
\hline \multicolumn{2}{|c|}{ Feb } & 0.038 & 0.036 & 0.00 & 0.00 & 0.218 & 0.219 & 0.026 & 0.031 & 0.034 & 0.037 & 0.056 & 0.051 & 0.965 & 0.875 \\
\hline \multicolumn{2}{|c|}{ Mar } & 0.051 & 0.054 & 0.00 & 0.00 & 0.256 & 0.239 & 0.028 & 0.032 & 0.027 & 0.033 & 0.052 & 0.043 & 0.992 & 0.911 \\
\hline \multicolumn{2}{|c|}{ Apr } & 0.035 & 0.048 & 0.00 & 0.00 & 0.254 & 0.241 & 0.037 & 0.042 & 0.028 & 0.029 & 0.048 & 0.039 & 0.985 & 0.889 \\
\hline \multicolumn{2}{|c|}{ May } & 0.054 & 0.049 & 0.00 & 0.00 & 0.234 & 0.242 & 0.032 & 0.038 & 0.034 & 0.041 & 0.043 & 0.036 & 0.993 & 0.911 \\
\hline \multicolumn{2}{|c|}{ Jun } & 0.037 & 0.047 & 0.00 & 0.00 & 0.241 & 0.251 & 0.038 & 0.039 & 0.032 & 0.039 & 0.049 & 0.047 & 0.997 & 0.924 \\
\hline \multicolumn{2}{|c|}{ Jul } & 0.038 & 0.041 & 0.00 & 0.00 & 0.234 & 0.236 & 0.035 & 0.031 & 0.037 & 0.045 & 0.042 & 0.038 & 1.087 & 1.002 \\
\hline \multicolumn{2}{|c|}{ Aug } & 0.047 & 0.055 & 0.00 & 0.00 & 0.265 & 0.253 & 0.029 & 0.037 & 0.031 & 0.042 & 0.048 & 0.044 & 0.967 & 0.907 \\
\hline \multicolumn{2}{|c|}{ Sep } & 0.045 & 0.051 & 0.00 & 0.00 & 0.251 & 0.245 & 0.034 & 0.039 & 0.029 & 0.034 & 0.052 & 0.046 & 0.939 & 0.912 \\
\hline \multicolumn{2}{|c|}{ Oct } & 0.037 & 0.043 & 0.00 & 0.00 & 0.265 & 0.250 & 0.032 & 0.037 & 0.035 & 0.043 & 0.054 & 0.048 & 1.073 & 0.995 \\
\hline \multicolumn{2}{|c|}{$\begin{array}{l}\text { Average } \\
\pm \text { SD }\end{array}$} & $\begin{array}{l}0.043 \\
\pm 0.006 \\
\end{array}$ & $\begin{array}{l}0.048 \\
\pm 0.006 \\
\end{array}$ & $\begin{array}{l}\mathbf{0 . 0 0} \\
\pm \mathbf{0 . 0 0} \\
\end{array}$ & $\begin{array}{l}\mathbf{0 . 0 0} \\
\pm 0.00 \\
\end{array}$ & $\begin{array}{l}0.245 \\
\pm 0.014\end{array}$ & $\begin{array}{l}0.239 \\
\pm 0.010 \\
\end{array}$ & $\begin{array}{l}\mathbf{0 . 0 3 3} \\
\pm 0.004 \\
\end{array}$ & $\begin{array}{l}\mathbf{0 . 0 3 7} \\
\pm \mathbf{0 . 0 0 5} \\
\end{array}$ & $\begin{array}{l}\mathbf{0 . 0 3 2} \\
\pm 0.004 \\
\end{array}$ & $\begin{array}{l}\mathbf{0 . 0 3 8} \\
\pm \mathbf{0 . 0 0 5} \\
\end{array}$ & $\begin{array}{l}0.050 \\
\pm 0.005 \\
\end{array}$ & $\begin{array}{l}0.044 \\
\pm 0.005\end{array}$ & $\begin{array}{l}1.007 \\
\pm 0.048 \\
\end{array}$ & $\begin{array}{l}0.936 \\
\pm 0.046 \\
\end{array}$ \\
\hline
\end{tabular}

158

Environment Conservation Journal 


\section{Assessment of the soil quality of Haridwar Uttarakhand India}

in the soil of the Control site (SS1) while maximum average $\mathrm{pH}$ was found $8.14 \pm 0.24$ for the second year in the soil of the Industrial area (SS3) and the average values of $\mathrm{pH}$ was found $7.60 \pm 0.09$, $7.66 \pm 0.13,8.04 \pm 0.15$, and $7.86 \pm 0.11$ at $\mathrm{SS} 1, \mathrm{SS} 2$, SS3, and SS4 respectively. $\mathrm{pH}$ was found maximum at the industrial area site $(8.04 \pm 0.15)$ while minimum was found at the control site (7.60 \pm 0.09$). \mathrm{pH}$ was found higher than the available literature (Shah, 2014; Kumar et al., 2020). A high range of $\mathrm{pH}$ was observed by Shah (2014) and Bharti and Kamboj (2018). During the whole study period, minimum average moisture was found $12.95 \% \pm 1.36$ for the second year in the soil of the Control site (SS1) while maximum average moisture was found $28.63 \% \pm 1.10$ for the second year in the soil of the Industrial area (SS3) and the average values of moisture was found $13.05 \pm 0.13, \quad 17.66 \pm 0.24, \quad 28.39 \pm 0.35, \quad$ and $23.88 \pm 0.61$ at SS1, SS2, SS3, and SS4 respectively. Bharti and Kamboj (2018) studied the soil moisture in 2018 and found less soil moisture. High moisture in the industrial area may be due to the covering of the ground surface and addition of solid waste which increase the moisture due to leachate.

During the whole study period, minimum average porosity was found $36.62 \% \pm 1.55$ for the first year in the soil of the Control site (SS1) while maximum average porosity was found $50.32 \% \pm 1.30$ for the first year in the soil of the Agricultural area (SS4) and the average values of porosity was found $37.56 \pm 1.33, \quad 38.50 \pm 0.86, \quad 48.32 \pm 0.83, \quad$ and $49.03 \pm 1.83$ at SS1, SS2, SS3, and SS4 respectively. Soil porosity was found maximum at the agricultural site $(50.32 \% \pm 1.30)$ while minimum was found at the control site $(36.62 \% \pm 1.55)$. In literature no study was found regarding porosity change in the soil. High porosity at agricultural site is the indicator of good soil heath at that site during the study period. During the whole study period, minimum average water holding capacity was found $35.60 \% \pm 1.70$ for the second year in the soil of the Control site (SS1) while maximum average water holding capacity was found $44.57 \% \pm 1.13$ for the second year in the soil of the Industrial area (SS3) and the average values of water holding capacity was found $36.22 \pm 0.87,40.19 \pm 1.02$, $43.58 \pm 1.41$, and $41.74 \pm 1.20$ at SS1, SS2, SS3, and SS4 respectively. Water holding capacity (WHC) was found maximum at the industrial area site
$(43.58 \pm 1.41)$ while minimum was found at the control site $(36.22 \pm 0.87)$. Negligible variation was found from results obtained by Bharti and Kamboj (2018).

During the whole study period, minimum average conductivity was found $0.192 \mu \mathrm{Mhos} / \mathrm{cm} \pm 0.028$ for the first year in the soil of the Residential area (SS2) while maximum average conductivity was found $0.425 \mu \mathrm{Mhos} / \mathrm{cm} \pm 0.063$ for the second year in the soil of the Industrial area (SS3) and the average values of conductivity was found $0.25 \pm 0.01,0.20 \pm 0.01,0.40 \pm 0.03$, and $0.37 \pm 0.00$ at $\mathrm{SS} 1, \mathrm{SS} 2, \mathrm{SS} 3$, and SS4 respectively. Electrical Conductivity (EC) was found maximum at the industrial area site $(0.40 \pm 0.03)$ while minimum was found at the control site $(0.20 \pm 0.01)$. Soil contamination reduces the conductivity of the soil may be due to binding of ions with pollutant. In all the recent literature a high conductivity was observed. During the whole study period, minimum average chloride was found $16.47 \mathrm{mg} / \mathrm{gm} \pm 1.09$ for the first year in the soil of the Control site (SS1) while maximum average chloride was found $54.48 \mathrm{mg} / \mathrm{gm} \pm 1.19$ for the second year in the soil of the Industrial area (SS3) and the average values of chloride was found $16.67 \pm 0.28, \quad 17.20 \pm 0.08$, $53.97 \pm 0.73$, and $20.94 \pm 3.17$ at SS1, SS2, SS3, and SS4 respectively. Chloride was found maximum at the industrial area site $(53.97 \pm 0.73)$ while minimum was found at the control site (16.67 \pm 0.28$)$. In literature no study was found regarding chloride change in the soil. Chlorides in the soil increase the salinity of the soil which results in the decreased fertility of the soil. During the whole study period, minimum average copper was found $0.031 \mathrm{mg} / \mathrm{gm} \pm 0.003$ for the first year in the soil of the Control site (SS1) while maximum average copper was found $0.077 \mathrm{mg} / \mathrm{gm} \pm 1.19$ for the second year in the soil of the Industrial area (SS3) and the average values of copper was found $0.03 \pm 0.00,0.05 \pm 0.00,0.07 \pm 0.00$, and $0.06 \pm 0.00$ at $\mathrm{SS} 1, \mathrm{SS} 2, \mathrm{SS} 3$, and SS4 respectively. Copper $(\mathrm{Cu})$ was found maximum at the industrial area site $(0.07 \pm 0.00)$ while minimum was found at the control site $(0.03 \pm 0.00)$ with an average value of $50.0 \mathrm{mg} / \mathrm{Kg}$. Copper was found in higher concentration during the study as compared to recent literature due to the impact of industrial discharge (Kumar et al., 2020; Shah, 2014; Kumar and Chopra, 2015). 
Table 5. Study of physicochemical parameters of Soil at industrial area site during 2013-2014

\begin{tabular}{|c|c|c|c|c|c|c|c|c|c|c|c|c|c|c|c|}
\hline \multirow{3}{*}{$\stackrel{3}{\stackrel{3}{E}}$} & \multirow{3}{*}{$\begin{array}{l}\text { Year } \\
\rightarrow\end{array}$} & \multicolumn{14}{|c|}{ Parameters } \\
\hline & & \multicolumn{2}{|c|}{ Temperature $\left({ }^{\circ} \mathrm{C}\right)$} & \multicolumn{2}{|l|}{$\mathbf{p H}$} & \multicolumn{2}{|c|}{ Soil Moisture (\%) } & \multicolumn{2}{|c|}{ Porosity (\%) } & \multicolumn{2}{|c|}{$\begin{array}{l}\text { Water Holding } \\
\text { Capacity (\%) }\end{array}$} & \multicolumn{2}{|c|}{$\begin{array}{l}\text { Conductivity } \\
(\mu \mathrm{Mhos} / \mathrm{cm})\end{array}$} & \multicolumn{2}{|c|}{ Chloride (mg/gm) } \\
\hline & & 2013 & 2014 & 2013 & 2014 & 2013 & 2014 & 2013 & 2014 & 2013 & 2014 & 2013 & 2014 & 2013 & 2014 \\
\hline \multicolumn{2}{|l|}{ Nov } & 21.0 & 21.1 & 7.8 & 7.8 & 26.12 & 26.56 & 47.5 & 49.2 & 42.5 & 44.6 & 0.354 & 0.371 & 52.90 & 52.81 \\
\hline \multicolumn{2}{|l|}{ Dec } & 20.7 & 21.5 & 7.9 & 8.3 & 27.41 & 27.23 & 48.9 & 49.4 & 41.3 & 43.2 & 0.336 & 0.348 & 52.56 & 52.78 \\
\hline \multicolumn{2}{|l|}{ Jan } & 21.5 & 21.4 & 7.6 & 8.1 & 27.62 & 27.94 & 45.4 & 47.3 & 41.3 & 44.1 & 0.346 & 0.351 & 52.14 & 53.35 \\
\hline \multicolumn{2}{|l|}{ Feb } & 20.9 & 20.7 & 8.1 & 8.4 & 27.74 & 28.17 & 46.4 & 48.2 & 42.1 & 45.2 & 0.314 & 0.373 & 52.67 & 54.02 \\
\hline \multicolumn{2}{|c|}{ Mar } & 21.5 & 21.4 & 7.5 & 7.9 & 28.03 & 28.94 & 48.3 & 49.5 & 41.7 & 42.6 & 0.348 & 0.379 & 52.36 & 54.62 \\
\hline \multicolumn{2}{|l|}{ Apr } & 21.9 & 22.0 & 7.9 & 8.1 & 29.11 & 29.57 & 49.2 & 49.0 & 42.4 & 44.7 & 0.351 & 0.384 & 53.04 & 55.13 \\
\hline \multicolumn{2}{|c|}{ May } & 22.2 & 22.5 & 7.8 & 7.8 & 29.42 & 29.91 & 47.4 & 48.7 & 42.1 & 43.8 & 0.403 & 0.498 & 53.89 & 55.86 \\
\hline \multicolumn{2}{|l|}{ Jun } & 22.5 & 23.6 & 7.8 & 7.9 & 29.91 & 30.12 & 47.6 & 49.2 & 41.6 & 43.7 & 0.362 & 0.514 & 54.46 & 56.55 \\
\hline \multicolumn{2}{|l|}{ Jul } & 21.9 & 22.0 & 8.4 & 8.5 & 28.84 & 29.71 & 47.7 & 48.5 & 43.4 & 46.2 & 0.435 & 0.471 & 54.88 & 55.68 \\
\hline \multicolumn{2}{|l|}{ Aug } & 21.9 & 21.6 & 8.2 & 8.4 & 28.43 & 28.96 & 48.6 & 49.2 & 44.4 & 45.1 & 0.516 & 0.484 & 54.64 & 54.71 \\
\hline \multicolumn{2}{|l|}{ Sep } & 21.4 & 21.4 & 8.0 & 8.2 & 27.86 & 28.37 & 46.4 & 48.2 & 43.6 & 46.2 & 0.414 & 0.478 & 54.18 & 54.41 \\
\hline \multicolumn{2}{|l|}{ Oct } & 21.0 & 21.8 & 8.1 & 8.3 & 27.16 & 28.08 & 49.4 & 50.4 & 44.6 & 45.5 & 0.416 & 0.451 & 53.71 & 53.89 \\
\hline \multicolumn{2}{|c|}{$\begin{array}{l}\text { Average } \\
\pm \text { SD }\end{array}$} & $\begin{array}{l}21.53 \\
\pm 0.56\end{array}$ & $\begin{array}{l}21.75 \\
\pm 0.74\end{array}$ & $\begin{array}{l}7.93 \\
\pm 0.25\end{array}$ & $\begin{array}{l}8.14 \\
\pm 0.24\end{array}$ & $\begin{array}{l}28.14 \\
\pm 1.06\end{array}$ & $\begin{array}{l}28.63 \\
\pm 1.10\end{array}$ & $\begin{array}{l}47.73 \\
\pm 1.23\end{array}$ & $\begin{array}{l}48.90 \\
\pm 0.79\end{array}$ & $\begin{array}{l}42.58 \\
\pm 1.15\end{array}$ & $\begin{array}{l}44.57 \\
\pm 1.13\end{array}$ & $\begin{array}{l}\mathbf{0 . 3 8 3} \\
\pm 0.056\end{array}$ & $\begin{array}{l}0.425 \\
\pm 0.063\end{array}$ & $\begin{array}{l}\mathbf{5 3 . 4 5} \\
\pm 0.96\end{array}$ & $\begin{array}{l}54.48 \\
\pm 1.19\end{array}$ \\
\hline
\end{tabular}

Table 6. Heavy metals analysis of Soil at industrial area site during 2013-2014

\begin{tabular}{|c|c|c|c|c|c|c|c|c|c|c|c|c|c|c|c|}
\hline \multirow{3}{*}{ 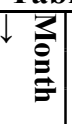 } & \multirow{3}{*}{$\begin{array}{l}\text { Year } \\
\rightarrow\end{array}$} & \multicolumn{14}{|c|}{ Parameters } \\
\hline & & \multicolumn{2}{|c|}{$\mathrm{Cu}(\mathrm{mg} / \mathrm{gm})$} & \multicolumn{2}{|c|}{ Cd(mg/gm) } & \multicolumn{2}{|c|}{ Mn (mg/gm) } & \multicolumn{2}{|c|}{ Ni (mg/gm) } & \multicolumn{2}{|c|}{$\mathrm{Pb}$ (mg/gm) } & \multicolumn{2}{|c|}{$\mathrm{Cr}^{+6}(\mathrm{mg} / \mathrm{gm})$} & \multicolumn{2}{|c|}{$\mathrm{Fe}(\mathrm{mg} / \mathrm{gm})$} \\
\hline & & 2013 & 2014 & 2013 & 2014 & 2013 & 2014 & 2013 & 2014 & 2013 & 2014 & 2013 & 2014 & 2013 & 2014 \\
\hline \multicolumn{2}{|c|}{ Nov } & 0.070 & 0.080 & 0.00 & 0.00 & 0.245 & 0.256 & 0.056 & 0.047 & 0.064 & 0.054 & 0.076 & 0.081 & 1.056 & 1.060 \\
\hline \multicolumn{2}{|l|}{ Dec } & 0.073 & 0.083 & 0.00 & 0.00 & 0.242 & 0.240 & 0.057 & 0.045 & 0.045 & 0.046 & 0.070 & 0.076 & 1.037 & 1.045 \\
\hline \multicolumn{2}{|l|}{ Jan } & 0.068 & 0.078 & 0.00 & 0.00 & 0.234 & 0.248 & 0.043 & 0.038 & 0.043 & 0.049 & 0.079 & 0.078 & 1.086 & 1.083 \\
\hline \multicolumn{2}{|l|}{ Feb } & 0.070 & 0.075 & 0.01 & 0.00 & 0.242 & 0.242 & 0.039 & 0.032 & 0.055 & 0.051 & 0.080 & 0.086 & 0.998 & 0.984 \\
\hline \multicolumn{2}{|l|}{ Mar } & 0.063 & 0.068 & 0.00 & 0.00 & 0.234 & 0.252 & 0.047 & 0.041 & 0.052 & 0.052 & 0.082 & 0.081 & 0.996 & 0.995 \\
\hline \multicolumn{2}{|l|}{ Apr } & 0.072 & 0.082 & 0.00 & 0.00 & 0.245 & 0.278 & 0.049 & 0.042 & 0.049 & 0.049 & 0.081 & 0.083 & 1.067 & 1.074 \\
\hline \multicolumn{2}{|l|}{ May } & 0.071 & 0.074 & 0.01 & 0.00 & 0.230 & 0.267 & 0.045 & 0.044 & 0.053 & 0.053 & 0.078 & 0.075 & 1.038 & 1.073 \\
\hline \multicolumn{2}{|l|}{ Jun } & 0.071 & 0.081 & 0.00 & 0.00 & 0.252 & 0.256 & 0.048 & 0.045 & 0.045 & 0.047 & 0.076 & 0.082 & 0.975 & 0.983 \\
\hline \multicolumn{2}{|l|}{ Jul } & 0.073 & 0.076 & 0.00 & 0.00 & 0.238 & 0.238 & 0.040 & 0.042 & 0.047 & 0.039 & 0.070 & 0.074 & 1.138 & 1.173 \\
\hline \multicolumn{2}{|l|}{ Aug } & 0.075 & 0.078 & 0.00 & 0.00 & 0.233 & 0.265 & 0.042 & 0.046 & 0.042 & 0.041 & 0.071 & 0.075 & 1.067 & 1.098 \\
\hline \multicolumn{2}{|l|}{ Sep } & 0.072 & 0.080 & 0.00 & 0.00 & 0.245 & 0.257 & 0.052 & 0.042 & 0.041 & 0.043 & 0.081 & 0.089 & 1.189 & 1.167 \\
\hline \multicolumn{2}{|l|}{ Oct } & 0.070 & 0.074 & 0.00 & 0.00 & 0.240 & 0.249 & 0.043 & 0.045 & 0.042 & 0.040 & 0.078 & 0.076 & 1.084 & 1.082 \\
\hline \multicolumn{2}{|c|}{$\begin{array}{l}\text { Average } \\
\pm \text { SD }\end{array}$} & $\begin{array}{l}0.071 \\
\pm 0.003 \\
\end{array}$ & $\begin{array}{l}\mathbf{0 . 0 7 7} \\
\pm 0.004 \\
\end{array}$ & $\begin{array}{l}0.002 \\
\pm 0.004 \\
\end{array}$ & $\begin{array}{l}0.00 \\
\pm 0.00 \\
\end{array}$ & $\begin{array}{l}0.240 \\
\pm 0.006 \\
\end{array}$ & $\begin{array}{l}0.254 \\
\pm 0.012 \\
\end{array}$ & $\begin{array}{l}0.047 \\
\pm 0.006 \\
\end{array}$ & $\begin{array}{l}0.042 \\
\pm 0.004 \\
\end{array}$ & $\begin{array}{l}0.048 \\
\pm 0.007 \\
\end{array}$ & $\begin{array}{l}0.047 \\
\pm 0.005 \\
\end{array}$ & $\begin{array}{l}\mathbf{0 . 0 7 7} \\
\pm \mathbf{0 . 0 0 4} \\
\end{array}$ & $\begin{array}{l}0.080 \\
\pm 0.005 \\
\end{array}$ & $\begin{array}{l}1.060 \\
\pm 0.061 \\
\end{array}$ & $\begin{array}{l}1.068 \\
\pm 0.062 \\
\end{array}$ \\
\hline
\end{tabular}


Table 7. Study of physicochemical parameters of Soil at Agricultural area site during 2013-2014

\begin{tabular}{|c|c|c|c|c|c|c|c|c|c|c|c|c|c|c|c|}
\hline \multirow{3}{*}{$\underset{\mathfrak{l}}{\stackrel{3}{\Xi}}$} & \multirow{3}{*}{$\begin{array}{l}\text { Year } \\
\rightarrow\end{array}$} & \multicolumn{14}{|c|}{ Parameters } \\
\hline & & \multicolumn{2}{|c|}{ Temperature $\left({ }^{\circ} \mathrm{C}\right)$} & \multicolumn{2}{|l|}{ pH } & \multicolumn{2}{|c|}{ Soil Moisture (\%) } & \multicolumn{2}{|c|}{ Porosity (\%) } & \multicolumn{2}{|c|}{$\begin{array}{l}\text { Water Holding } \\
\text { Capacity (\%) }\end{array}$} & \multicolumn{2}{|c|}{$\begin{array}{l}\text { Conductivity } \\
(\mu M h o s / \mathrm{cm})\end{array}$} & \multicolumn{2}{|c|}{ Chloride (mg/gm) } \\
\hline & & 2013 & 2014 & 2013 & 2014 & 2013 & 2014 & 2013 & 2014 & 2013 & 2014 & 2013 & 2014 & 2013 & 2014 \\
\hline \multicolumn{2}{|c|}{ Nov } & 19.2 & 21.0 & 7.9 & 7.8 & 23.01 & 24.21 & 49.5 & 47.5 & 42.5 & 41.2 & 0.343 & 0.344 & 20.17 & 22.90 \\
\hline \multicolumn{2}{|c|}{ Dec } & 18.9 & 19.8 & 7.8 & 7.9 & 22.98 & 23.86 & 50.9 & 48.9 & 40.4 & 39.4 & 0.332 & 0.325 & 20.98 & 22.96 \\
\hline \multicolumn{2}{|c|}{ Jan } & 18.6 & 19.6 & 7.5 & 7.6 & 22.81 & 23.63 & 48.4 & 45.4 & 41.3 & 38.6 & 0.334 & 0.334 & 21.56 & 23.47 \\
\hline \multicolumn{2}{|c|}{ Feb } & 19.0 & 20.1 & 8.0 & 8.1 & 23.52 & 24.48 & 49.4 & 46.4 & 42.1 & 39.1 & 0.304 & 0.302 & 21.90 & 23.88 \\
\hline \multicolumn{2}{|c|}{ Mar } & 19.2 & 20.3 & 7.4 & 7.5 & 23.69 & 24.72 & 52.3 & 48.3 & 41.7 & 41.1 & 0.312 & 0.332 & 19.11 & 22.36 \\
\hline \multicolumn{2}{|c|}{ Apr } & 19.2 & 20.6 & 7.3 & 7.9 & 23.84 & 24.91 & 51.2 & 49.2 & 42.4 & 41.5 & 0.332 & 0.334 & 19.54 & 22.67 \\
\hline \multicolumn{2}{|c|}{ May } & 20.5 & 21.6 & 7.6 & 7.8 & 23.96 & 25.16 & 49.4 & 47.4 & 42.1 & 39.8 & 0.378 & 0.387 & 19.80 & 22.91 \\
\hline \multicolumn{2}{|c|}{ Jun } & 21.7 & 22.8 & 7.5 & 7.8 & 24.15 & 25.39 & 49.6 & 47.6 & 41.6 & 41.8 & 0.356 & 0.356 & 20.07 & 23.16 \\
\hline \multicolumn{2}{|l|}{ Jul } & 21.4 & 22.5 & 8.2 & 8.4 & 24.06 & 24.61 & 49.7 & 47.7 & 43.4 & 40.7 & 0.429 & 0.428 & 20.62 & 23.76 \\
\hline \multicolumn{2}{|c|}{ Aug } & 21.0 & 22.1 & 8.0 & 8.2 & 23.69 & 24.34 & 51.6 & 48.6 & 44.4 & 42.6 & 0.498 & 0.502 & 20.97 & 23.87 \\
\hline \multicolumn{2}{|c|}{ Sep } & 20.7 & 21.6 & 8.2 & 8.0 & 23.15 & 23.36 & 49.4 & 46.4 & 43.6 & 43.4 & 0.400 & 0.402 & 20.41 & 23.56 \\
\hline \multicolumn{2}{|l|}{ Oct } & 20.1 & 21.2 & 8.0 & 8.1 & 22.55 & 23.04 & 52.4 & 49.4 & 45.3 & 41.5 & 0.389 & 0.400 & 19.84 & 22.61 \\
\hline \multicolumn{2}{|c|}{$\begin{array}{l}\text { Average } \\
\pm \text { SD } \\
\end{array}$} & $\begin{array}{l}19.96 \\
\pm 1.07 \\
\end{array}$ & $\begin{array}{l}21.14 \\
\pm 1.05 \\
\end{array}$ & \begin{tabular}{l|}
7.78 \\
\pm 0.31 \\
\end{tabular} & \begin{tabular}{l|l}
7.93 \\
\pm 0.25 \\
\end{tabular} & $\begin{array}{l}23.45 \\
\pm 0.53 \\
\end{array}$ & $\begin{array}{l}24.31 \\
\pm 0.72 \\
\end{array}$ & $\begin{array}{l}\mathbf{5 0 . 3 2} \\
\pm 1.30 \\
\end{array}$ & $\begin{array}{l}47.73 \\
\pm 1.23 \\
\end{array}$ & $\begin{array}{l}42.58 \\
\pm 1.15 \\
\end{array}$ & $\begin{array}{l}40.89 \\
\pm 1.44 \\
\end{array}$ & $\begin{array}{l}\mathbf{0 . 3 6 7} \\
\pm \mathbf{0 . 0 5 3} \\
\end{array}$ & $\begin{array}{l}0.371 \\
\pm 0.056 \\
\end{array}$ & $\begin{array}{l}18.70 \\
\pm 0.87 \\
\end{array}$ & $\begin{array}{l}23.18 \\
\pm 0.52 \\
\end{array}$ \\
\hline
\end{tabular}

Table 8. Heavy metals analysis of Soil at the Agricultural area site during 2013-2014.

\begin{tabular}{|c|c|c|c|c|c|c|c|c|c|c|c|c|c|c|c|}
\hline \multirow{3}{*}{$\underset{\downarrow}{\stackrel{3}{E}}$} & \multirow{3}{*}{$\begin{array}{l}\text { Year } \\
\rightarrow\end{array}$} & \multicolumn{14}{|c|}{ Parameters } \\
\hline & & \multicolumn{2}{|c|}{$\mathrm{Cu}$ (mg/gm) } & \multicolumn{2}{|c|}{ Cd (mg/gm) } & \multicolumn{2}{|c|}{ Mn (mg/gm) } & \multicolumn{2}{|c|}{$\mathrm{Ni}(\mathrm{mg} / \mathrm{gm})$} & \multicolumn{2}{|c|}{$\mathrm{Pb}$ (mg/gm) } & \multicolumn{2}{|c|}{$\mathrm{Cr}^{+6}(\mathrm{mg} / \mathrm{gm})$} & \multicolumn{2}{|c|}{$\mathrm{Fe}$ (mg/gm) } \\
\hline & & 2013 & 2014 & 2013 & 2014 & 2013 & 2014 & 2013 & 2014 & 2013 & 2014 & 2013 & 2014 & 2013 & 2014 \\
\hline \multicolumn{2}{|c|}{ Nov } & 0.055 & 0.065 & 0.00 & 0.00 & 0.201 & 0.244 & 0.048 & 0.036 & 0.061 & 0.034 & 0.071 & 0.065 & 0.856 & 0.960 \\
\hline \multicolumn{2}{|c|}{ Dec } & 0.069 & 0.064 & 0.00 & 0.00 & 0.212 & 0.234 & 0.038 & 0.035 & 0.040 & 0.037 & 0.067 & 0.066 & 0.736 & 0.945 \\
\hline \multicolumn{2}{|c|}{ Jan } & 0.060 & 0.068 & 0.01 & 0.00 & 0.202 & 0.233 & 0.029 & 0.028 & 0.042 & 0.038 & 0.068 & 0.058 & 0.787 & 0.883 \\
\hline \multicolumn{2}{|c|}{ Feb } & 0.049 & 0.056 & 0.00 & 0.00 & 0.212 & 0.226 & 0.020 & 0.023 & 0.051 & 0.043 & 0.067 & 0.074 & 0.897 & 0.884 \\
\hline \multicolumn{2}{|c|}{ Mar } & 0.044 & 0.054 & 0.00 & 0.00 & 0.219 & 0.226 & 0.031 & 0.031 & 0.048 & 0.041 & 0.065 & 0.068 & 0.796 & 0.895 \\
\hline \multicolumn{2}{|c|}{ Apr } & 0.059 & 0.076 & 0.00 & 0.00 & 0.216 & 0.237 & 0.028 & 0.032 & 0.043 & 0.040 & 0.066 & 0.069 & 0.867 & 0.874 \\
\hline \multicolumn{2}{|c|}{ May } & 0.049 & 0.066 & 0.00 & 0.00 & 0.229 & 0.256 & 0.023 & 0.034 & 0.050 & 0.042 & 0.056 & 0.065 & 0.838 & 0.973 \\
\hline \multicolumn{2}{|c|}{ Jun } & 0.060 & 0.076 & 0.00 & 0.00 & 0.224 & 0.243 & 0.027 & 0.035 & 0.042 & 0.041 & 0.065 & 0.075 & 0.675 & 0.783 \\
\hline \multicolumn{2}{|c|}{ Jul } & 0.045 & 0.056 & 0.00 & 0.00 & 0.217 & 0.227 & 0.038 & 0.032 & 0.041 & 0.023 & 0.045 & 0.064 & 0.738 & 0.973 \\
\hline \multicolumn{2}{|c|}{ Aug } & 0.054 & 0.048 & 0.00 & 0.00 & 0.203 & 0.222 & 0.033 & 0.036 & 0.038 & 0.034 & 0.034 & 0.062 & 0.567 & 0.998 \\
\hline \multicolumn{2}{|c|}{ Sep } & 0.067 & 0.061 & 0.00 & 0.00 & 0.198 & 0.243 & 0.025 & 0.032 & 0.040 & 0.032 & 0.056 & 0.079 & 0.689 & 1.067 \\
\hline \multicolumn{2}{|c|}{ Oct } & 0.044 & 0.056 & 0.00 & 0.00 & 0.189 & 0.225 & 0.021 & 0.035 & 0.032 & 0.031 & 0.056 & 0.064 & 0.884 & 0.882 \\
\hline \multicolumn{2}{|c|}{$\begin{array}{l}\text { Average } \\
\pm \text { SD }\end{array}$} & $\begin{array}{l}\mathbf{0 . 0 5 5} \\
\pm 0.009\end{array}$ & $\begin{array}{l}0.062 \\
\pm 0.008\end{array}$ & $\begin{array}{l}0.00 \\
\pm 0.002\end{array}$ & $\begin{array}{l}\mathbf{0 . 0 0} \\
\pm 0.00\end{array}$ & $\begin{array}{l}0.210 \\
\pm 0.01\end{array}$ & $\begin{array}{l}0.235 \\
\pm 0.01\end{array}$ & $\begin{array}{l}\mathbf{0 . 0 3 0} \\
\pm 0.008\end{array}$ & $\begin{array}{l}\mathbf{0 . 0 3 2} \\
\pm 0.004\end{array}$ & $\begin{array}{l}0.044 \\
\pm 0.008\end{array}$ & $\begin{array}{l}\mathbf{0 . 0 3 6} \\
\pm 0.004\end{array}$ & $\begin{array}{l}0.059 \\
\pm 0.011\end{array}$ & $\begin{array}{l}0.067 \\
\pm 0.006\end{array}$ & $\begin{array}{l}0.778 \\
\pm 0.099\end{array}$ & $\begin{array}{l}0.926 \\
\pm 0.074\end{array}$ \\
\hline
\end{tabular}

161

Environment Conservation Journal 


\section{Bhardwaj et al.}

During the whole study period, minimum average cadmium was found $0.00 \mathrm{mg} / \mathrm{gm} \pm 0.00$ for the second year in the soil of all the sites while maximum average cadmium was found 0.01 $\mathrm{mg} / \mathrm{gm} \pm 0.00$ for the first year in the soil of the SS3 and SS4 and the average values of cadmium was found $0.00 \pm 0.00$ at SS1, SS2, SS3, and SS4 respectively. During the whole study period, minimum average manganese was found $0.210 \mathrm{mg} / \mathrm{gm} \pm 0.01$ for the first year in the soil of the Agricultural area (SS4) while maximum average manganese was found $0.254 \mathrm{mg} / \mathrm{gm} \pm 0.012$ for the second year in the soil of the Industrial area (SS3) and the average values of manganese was found $0.24 \pm 0.00,0.24 \pm 0.00,0.25 \pm 0.01$, and $0.22 \pm 0.02$ at SS1, SS2, SS3, and SS4 respectively. Manganese $(\mathrm{Mn})$ was found maximum at the industrial area site $(0.25 \pm 0.01)$ while minimum was found at the control site $(0.22 \pm 0.02)$. Mn value was also found in higher concentration during the study period form all the recent observed literature clearly showing the impact of industrial solid and liquid waste dumping.

Nickel is an essential micronutrient for thwe growth of plants. Nickel absorption takes place through active and passive diffiusion and endocytosis (Ahmad and Ashraf, 2011). During the whole study period, minimum average nickel was found $0.030 \mathrm{mg} / \mathrm{gm} \pm 0.005$ for the first year in the soil of the Control site (SS1) and Agricultural area (SS4) while maximum average nickel was found $0.047 \mathrm{mg} / \mathrm{gm} \pm 0.006$ for the first year in the soil of the Industrial area (SS3) and the average values of nickel was found $0.03 \pm 0.00,0.04 \pm 0.00,0.04 \pm 0.00$, and $0.03 \pm 0.00$ at SS1, SS2, SS3, and SS4 respectively. Nickel was present in less quantity as at the study no source of nicekl was observed in the study area. Maxiumu quantity of the nickel was found at Agricultural area (SS4) which is a bad indicator for the agicultural production as the excess quantity of nickel affects the absorption of nutrientrs by plants (Ahmad and Ashraf, 2011). During the whole study period, minimum average lead was found $0.030 \mathrm{mg} / \mathrm{gm} \pm 0.002$ for the first year in the soil of the Control site (SS1) while maximum average lead was found $0.048 \mathrm{mg} / \mathrm{gm} \pm 0.007$ for the first year in the soil of the Industrial area (SS3) and the average values of lead was found $0.03 \pm 0.00,0.04 \pm 0.00,0.05 \pm 0.00$, and $0.04 \pm 0.01$ at $\mathrm{SS} 1, \mathrm{SS} 2, \mathrm{SS} 3$, and SS4 respectively. Toxicity of chromium depends on its oxidation states. $\mathrm{Cr}^{+6}$ is more dangerous and mobile than $\mathrm{Cr}^{+3}$. During the whole study period, minimum average chromium was found $0.033 \mathrm{mg} / \mathrm{gm} \pm 0.005$ for the first year in the soil of the Control site (SS1) while maximum average chromium was found $0.080 \mathrm{mg} / \mathrm{gm} \pm 0.005$ for the second year in the soil of the Industrial area (SS3) and the average values of chromium was found $0.03 \pm 0.00,0.05 \pm 0.00,0.08 \pm 0.00$, and $0.06 \pm 0.01$ at SS1, SS2, SS3, and SS4 respectively. Maximum values of $\mathrm{Cr}$ was found in industrial area may be due to dumping of industrial area waste in the soil (Oliveira, 2012). Toxicity of $\mathrm{Cr}$ may be reduced with the help of oxidation with iron, vanadium, sulphydes, and organic materials (Cary, 1982).

During the whole study period, minimum average iron was found $00.778 \mathrm{mg} / \mathrm{gm} \pm 0.99$ for the first year in the soil of the Agricultural area site (SS4) while maximum average iron was found $1.931 \mathrm{mg} / \mathrm{gm} \pm 0.47$ for the second year in the soil of the Residential area (SS2) and the average values of iron was found $1.92 \pm 0.01,0.97 \pm 0.05,1.06 \pm 0.01$, and $0.85 \pm 0.10$ at SS1, SS2, SS3, and SS4 respectively. Iron $(\mathrm{Fe})$ was observed in less concentration during the study period as compared to the results obtained by Kumar et al. (2020) and Shah (2014) while higher concentration was observed as compared to the results obtained by Kumar and Chopra (2015). Minimum concentration at agricultural area may be due to utilisation of iron by the crops as it is essential component of plant growth and required for the functioning of various enzymes.

\section{Conclusion}

The physicochemical properties of the soil studied were observed affected may be due to different anthropogenic activities. During the course of study an increasing trend in all the parameters was observed. Although all the parameters studied during the study were found within the prescribed limit but the day to day increase in the anthropogenic activities is a threat to the health of soil. When the results were compared with the results obtained in recent literature, most of the parameters were found in higher concentration but a reduction in all the parameters was observed. In agricultural fields an increasing trend of heavy 
Assessment of the soil quality of Haridwar Uttarakhand India

Table 9. Showing the comparison of results obtained at different locations

\begin{tabular}{|l|l|l|l|l|l|}
\hline $\begin{array}{l}\text { Parameters/ } \\
\text { Study site }\end{array}$ & $\begin{array}{l}\text { Control site } \\
\text { (Forest area) }\end{array}$ & $\begin{array}{l}\text { Industrial area } \\
\text { site }\end{array}$ & $\begin{array}{l}\text { Residential area } \\
\text { site }\end{array}$ & $\begin{array}{l}\text { Agricultural area } \\
\text { site }\end{array}$ & $\begin{array}{l}\text { AVG (in } \\
\text { mg/gm) }\end{array}$ \\
\hline Temperature (o-C) & $16.63 \pm 0.30$ & $18.93 \pm 0.11$ & $21.64 \pm 0.16$ & $20.55 \pm 0.83$ & 19.43 \\
\hline pH & $7.60 \pm 0.09$ & $7.66 \pm 0.13$ & $8.04 \pm 0.15$ & $7.86 \pm 0.11$ & 7.79 \\
\hline Soil Moisture (\%) & $13.05 \pm 0.13$ & $17.66 \pm 0.24$ & $28.39 \pm 0.35$ & $23.88 \pm 0.61$ & 20.67 \\
\hline Porosity (\%) & $37.56 \pm 1.33$ & $38.50 \pm 0.86$ & $48.32 \pm 0.83$ & $49.03 \pm 1.83$ & 42.81 \\
\hline $\begin{array}{l}\text { Water Holding } \\
\text { Capacity (\%) }\end{array}$ & $36.22 \pm 0.87$ & $40.19 \pm 1.02$ & $43.58 \pm 1.41$ & $41.74 \pm 1.20$ & 38.42 \\
\hline $\begin{array}{l}\text { Conductivity } \\
\text { ( } \boldsymbol{\mu M h o s / c m ) ~}\end{array}$ & $0.25 \pm 0.01$ & $0.20 \pm 0.01$ & $0.40 \pm 0.03$ & $0.37 \pm 0.00$ & 0.31 \\
\hline Chloride (mg/gm) & $16.67 \pm 0.28$ & $17.20 \pm 0.08$ & $53.97 \pm 0.73$ & $20.94 \pm 3.17$ & 27.41 \\
\hline Cu (mg/gm) & $0.03 \pm 0.00$ & $0.05 \pm 0.00$ & $0.07 \pm 0.00$ & $0.06 \pm 0.00$ & 0.05 \\
\hline Cd (mg/gm) & $0.00 \pm 0.00$ & $0.00 \pm 0.00$ & $0.00 \pm 0.00$ & $0.00 \pm 0.00$ & 0.00 \\
\hline Mn (mg/gm) & $0.24 \pm 0.00$ & $0.24 \pm 0.00$ & $0.25 \pm 0.01$ & $0.22 \pm 0.02$ & 0.24 \\
\hline Ni (mg/gm) & $0.03 \pm 0.00$ & $0.04 \pm 0.00$ & $0.04 \pm 0.00$ & $0.03 \pm 0.00$ & 0.04 \\
\hline Pb (mg/gm) & $0.03 \pm 0.00$ & $0.04 \pm 0.00$ & $0.05 \pm 0.00$ & $0.04 \pm 0.01$ & 0.04 \\
\hline Cr (mg/gm) & $0.03 \pm 0.00$ & $0.05 \pm 0.00$ & $0.08 \pm 0.00$ & $0.06 \pm 0.01$ & 0.06 \\
\hline Fe (mg/gm) & $1.92 \pm 0.01$ & $0.97 \pm 0.05$ & $1.06 \pm 0.01$ & $0.85 \pm 0.10$ & 1.21 \\
\hline
\end{tabular}

metals was observed in recent literature showing a threat to agricultural ecosystem. Therefore continuous monitoring of agricultural land around dumping sites is also required to safeguard the health of people as the translocation of all these pollutants in vegetables can affect the heath of peoples. There is need to increase the awareness among the farmers to make them aware about the use of required quantity of irrigation form the wastewater in the fields.

\section{References}

Ahmad, M. S. A. and Ashraf, M. 2011. Essential roles and hazardous effects of nickel in plants. Reviews of Environmental Contamination and Toxicology, 214: 125-67. doi: 10.1007/978-1-4614-0668-6_6.

Bharti, M. and Kamboj, N. 2018. Impact of different land uses on soil characteristics in Ranipur rao Watershed in Haridwar district, Uttarakhand. International Journal of Creative Research Thoughts (IJCRT), 6(1): 1147-1154.

Bougnom, B. P., Thiele-Bruhn, S., Ricci, V., Zongo, C. and Piddock, L. J. V. 2020. Raw wastewater irrigation for urban agriculture in three African cities increases the abundance of transferable antibiotic resistance genes in soil, including those encoding extended spectrum $\beta$ lactamases (ESBLs). Science of the Total Environment, 698: 134201 .

Cary, E. E. 1982. Chromium in air, soils, and natural waters in Biological and Environmental Aspects of Chromium, S.
Langard, Ed., pp. 49-63, Elsevier Biomedical, New York, NY, USA,

Courault, D., Albert, I., Perelle, S., Fraisse, A., Renault, P., Salemkour, A. and Amato, P. 2017. Assessment and risk modeling of airborne enteric viruses emitted fromwastewater reused for irrigation. Science of the Total Environment, 592: 512-526.

http://agricoop.nic.in/sites/default/files/haridwar.pdf GOVT web)

(UK

James, A., Thomas, T. and Kumar, S. 2014. Effect of flyash on the physiochemical properties of soil health and mustard crop. International Journal of Agricultural Sciences, 10: 453-458.

Jiang, Y., Zang, G., Zou, D., Qin, Y. and Liang, W. J. 2009. Profile distribution of micronutrients in a aquic brown soil as affected by land use. Journal of Plant Soil Environment, 155(11): 468-476.

Kumar, V. and Chopra, A. K. 2015. Heavy Metals Accumulation in Soil and Agricultural Crops Grown in the Province of Asahi India Glass Ltd., Haridwar (Uttarakhand), India. Advances in Crop Science and Technology, 4:1.

Kumar, V., Singh, J. and Kumar, P. 2019. Heavy metal uptake by water lettuce (Pistia stratiotes L.) from paper mill effluent (PME): experimental and prediction modeling studies. Environmental Science and Pollution Research. https://doi. org/10.1007/s11356-019-04766-2.

Kumar, V., Thakur, R. K. and Kumar, P. 2020. Predicting heavy metals uptake by spinach (Spinacia oleracea) grown 
in integrated industrial wastewater irrigated soils of Haridwar, India. Environmental Monitoring Assessment, 192:709.

Oliveira, H. 2012. Chromium as an Environmental Pollutant: Insights on Induced Plant Toxicity. Journal of Botany, Article ID 375843, 8 pages doi:10.1155/2012/375843

Papendick, S. B. and Parr, J. F. 1992. Soil Quality: Attributes and Relationship to Alternative and Sustainable Agriculture. American Journal Alter. Agri., 7(1-2): 2-3.

Raja, R., Nayak, A. K., Shukla, A. K. et. al. 2015. Impairment of soil health due to fly ash-fugitive dust deposition from coal-fired thermal power plants. Environmental Monitoring and Assessment, 187: 679.

Sarwar, T., Shahid, M., Khalid, S., Shah, A. H., Ahmad, N. and Naeem, M. A. 2019. Quantification and risk assessment of heavy metal build-up in soil-plant system after irrigation with untreated city wastewater in Vehari, Pakistan. Environmental Geochemistry and Health, 1-17.

Shah, S. (2014). Impact of Natural hazard and human interference on river Ganga basin in the neighhbourhood of Kankhal Uttarakhand. A thesis submitted to Department of Geography university of Calcutta. http://hdl.handle.net/10603/163789.

Singh, D., Chhankar, P. K. and Pandey, R. N. 1999. Soil, Plant and Water analysis a method manual. IARI, New Delhi.

Trivedi, R. K. and Goel, P. K. 1986. Chemical and Biological methods for water pollution studies karad. Environmental publication, 1-251.

Zhang, Y. and Shen, Y. 2019. Wastewater irrigation: past, present, and future. Wiley Interdisciplinary Reviews Water, 6(3): e1234. 